\title{
Effect of different fertilizing and farming systems in annual medic (Medicago scutellata 'Robinson') on soil organic matter and nutrients status
}

\author{
G. SHABANI ${ }^{*}$, M. R. CHAICHI ${ }^{2}$, M. R. ARDAKANI ${ }^{3}$, J. K. FRIEDEL ${ }^{4}$, K. KHAVAZI $^{5}$
}

Received Janury 19, 2016; accepted Janury 01, 2017.

Delo je prispelo 19. januarja 2016, sprejeto 01. januarja 2017.

\begin{abstract}
This experiment was conducted to study the effect of different fertilizing and farming systems in annual medic (Medicago scutellata 'Robinson') on soil organic matter and nutrients status. Fertilizing systems consisted of control (no fertilizer), chemical fertilizer, biological fertilizer and integrated fertilizers (different combinations of chemical and biological fertilizing systems). The farming systems included irrigated and dry-farming systems. The experiment was conducted in two experimental sites with diverse climatic and soil conditions in Kermanshah province, Iran, during 2009 growing season. The highest amount of soil organic matter of $1.28 \%$ was observed in integrated fertilizing system of nitrogen-fixing bacteria + phosphorus-solubilizing bacteria. Most of the nitrogen applied through chemical fertilizers was leached out of the plant access, however, application of integrated fertilizer resulted in increasing the concentration of nitrogen in soil because of its slow release and efficient utilization by plants. According to the results of this study it was concluded that the integrated fertilizing system was more successful in dry farming compared to other fertilizing systems.
\end{abstract}

Key words: annual medic; fertilizing system; farming system; soil nutrients; soil organic matter; climatic conditions

\section{IZVLEČEK}

UČINEK RAZLIČNIH NAČINOV GNOJENJA IN
KMETOVANJA NA VSEBNOST ORGANSKE SNOVI
IN HRANIL V TLEH V POSEVKU ŠČITASTOPLODNE
METELJKE (Medicago scutellata 'Robinson')

V poskusu je bil preučevan učinek različnih načinov gnojenja in kmetovanja na vsebnost organske snovi in hranil $\mathrm{v}$ tleh $\mathrm{v}$ posevku šcitastoplodne meteljke (Medicago scutellata 'Robinson'). Gnojenja so obsegala kontrolo (brez gnojil), mineralna gnojila, biološka gnojila in integrirano gnojenje (različne kombinacije mineralnih in bioloških gnojil). Načina kmetovanja sta bila kmetovanje $\mathrm{z}$ namakanjem in brez namakanja. Poskus je potekal na dveh poskusnih mestih, ki sta se razlikovali $\mathrm{v}$ talnih in podnebnih razmerah $\mathrm{v}$ provinci Kermanshah, Iran, v rastni sezoni 2009. Največja vsebnost organske snovi $\mathrm{v}$ tleh $(1.28 \%)$ je bila izmerjena pri integriranemu načinu gnojenja, $\mathrm{v}$ katerem so uporabili bakterije, ki vežejo zračni dušik in bakterije, ki sproščajo fosfor. Večina dušika, ki je bila dodana v obliki mineralnih gnojil, je bila izprana iz tal, pri integriranem gnojenju pa se je koncentracija dušika $\mathrm{v}$ tleh povečala zaradi počasnejšega sproščanja in učinkovitega privzema $\mathrm{v}$ rastline. Glede na izsledke te raziskave lahko zaključimo, da je integrirani način gnojenja uspešnejši pri kmetovanju brez namakanja $\mathrm{v}$ primerjavi z drugimi načini gnojenja.

Ključne besede: ščitastoplodna meteljka; način gnojenja; način kmetovanja; vsebnost hranil v tleh; organska snov tal; podnebne razmere

\footnotetext{
1 Young Researchers and Elite Club, Malayer Branch, Islamic Azad University, Malayer, Islamic Republic of Iran*correspponding author: bb1379@gmail.com

2 College of Agriculture, California State Polytechnic University, Pomona, USA

3 College of Agriculture and Natural Resources, Islamic Azad University, Karaj Branch, Iran

4 Department of Sustainable Agricultural Systems, University of Natural Resources and Life Sciences, Vienna, Austria

5 Soil and Water Research Institute, Iran
} 


\section{INTRODUCTION}

Sustainability in agricultural production systems is one of the main management goals. The application of chemical fertilizers has been the best way to increase crop production towards meeting the global demands of food production (Shahoo et al., 2013). Their adverse impacts on soil are not effectively compatible with the goals of the sustainable management of agro-ecosystems (Banerjee et al., 2011 and Garai et al., 2014, Mondal et al., 2015). On the other hand, biofertilizers known as the environmental-friendly fertilizers can contribute achieving these goals. However, an economic production, an optimization in fertilizer application, a proper use of pesticides, an increase in soil organic matter, and an environmentally safe production system are required.

It is well known that biofertilizers affect the nutrients uptake by plants in normal and harsh environments (Mal et al., 2013; Hariprasad and Niranjana, 2009; Kim et al. 2009). Employing biological fertilizers, such as symbiotic fungi coexisting with plant roots, phosphate solubilizing micro-organisms and vermicompost in agriculture, not only increase the population and activity of beneficial soil micro-organisms, but also improve the growth and yield of crops (Mondal et al., 2015).

There are also some evidence that dual inoculation of medic under the phosphorus deficiency conditions led to increased mass, consolidation of $\mathrm{N}_{2}$ and phosphorus content in plants at different levels compared to mono inoculation (Starcheva et al., 2008). Dual inoculation of vesicular-arbuscular mycorrhizal (VAM) and phosphate-solubilizing bacteria (PSB) enhanced plant growth and development compared to sole inoculation with each of these micro-organisms. Increased plant growth and $\mathrm{P}$ uptake has been reported by many researchers in several different crop species (Shabani et al., 2015).

With biological fertilizers utilization, it can be postulated that the nitrogen availability for plants will increase providing the suitable growth conditions and so a better quality yields will be expected (Duarah et al., 2011). Combining the biological fertilizer with urea under a suitable irrigation conditions can lead to increased forage quality. Heretofore, mainly the effect of biological fertilizers on the uptake and storage of nitrogen and phosphorus in plants has been studied, however, the effect of biological fertilizers on conservation and utilization of micronutrient elements as well as soil organic matter has been less considered. In general, the results of conducted studies have shown that the application of biological fertilizers had positive effects in terms of quantity, and quality of yield in different crops.

The main goal of this research was to understand how the farming (irrigated and dry farming) and fertilizing systems interaction affect the soil organic matter, micronutrient uptake and annual medic forage yield. We tried to address the following questions:

- What is the best combination of fertilizer and farming system to improve soil organic matter?

- How the soil nutrients are affected by the fertilizer and farming system?

- Does the application of biofertilizers (symbiosis bacteria and mycorrhiza) result in higher yield and quality of annual medic forage?

\section{MATERIALS AND METHODS}

The experiment was conducted in two locations: 1 . Sararood Dryland Farming Research Station with the geographic longitude of 47', 20' and latitude of 34 ', 20' with an elevation of 1351 meters above the sea level, and 2. Mahidasht Soil Fertility Research Station with the geographic longitude of
46", 50' and latitude of 24", 16' with elevation of 1380 meters above the sea level, during 2009 growing season. Some physical and chemical characteristics of soil and climatic information of two experimental sites are shown in Tables 1 and 2. 
Table 1: Selected physical and chemical characteristics of soil $(0-30 \mathrm{~cm}$ depth) in two experimental sites

\begin{tabular}{lrr}
\hline \multirow{2}{*}{ Characteristic } & Experimental Stations \\
\cline { 2 - 3 } & Sararood & Mahidasht \\
\hline $\mathrm{pH}$ & 7.68 & 7.93 \\
Dissolved solids (EC.103) & 30.0 & 55.0 \\
Organic carbon (\%) & 0.31 & 0.62 \\
$\mathrm{CaCO}_{3}(\%)$ & 30 & 28 \\
Olsen phosphorous $\left(\mathrm{mg} \mathrm{kg}^{-1}\right)$ & 8.00 & 9.40 \\
Available potassium $\left(\mathrm{mg} \mathrm{kg}^{-1}\right)$ & 530 & 430 \\
DTPA extractable $\mathrm{Zn}\left(\mathrm{mg} \mathrm{kg}^{-1}\right)$ & 0.38 & 1.56 \\
DTPA extractable Cu $\left(\mathrm{mg} \mathrm{kg}^{-1}\right)$ & 0.70 & 1.40 \\
DTPA extractable Fe $\left(\mathrm{mg} \mathrm{kg}^{-1}\right)$ & 2.00 & 4.76 \\
DTPA extractable $\mathrm{Mn}\left(\mathrm{mg} \mathrm{kg}^{-1}\right)$ & 2.42 & 3.78 \\
Soil texture & Loamy silt & Loamy clay \\
\hline
\end{tabular}

Table 2: Mean precipitation and temperature during annual medic growing season in two experimental sites

\begin{tabular}{|c|c|c|c|c|}
\hline \multirow{2}{*}{ Month } & \multicolumn{2}{|c|}{ Average precipitation $(\mathrm{mm})$} & \multicolumn{2}{|c|}{ Average temperature $\left({ }^{\circ} \mathrm{C}\right)$} \\
\hline & Sararood & Mahidasht & Sararood & Mahidasht \\
\hline Feb. & 18.3 & 21.2 & 7.3 & 6.4 \\
\hline Mar. & 36.1 & 71.8 & 9.4 & 8.0 \\
\hline Apr. & 15.2 & 12.4 & 16.2 & 14 \\
\hline May & 0.2 & 0.9 & 22.7 & 19.7 \\
\hline Jun. & 0 & 0 & 26.5 & 24.0 \\
\hline
\end{tabular}

The experimental sites at both locations were kept as fallow in the year preceding the experiment. The experiment was conducted as randomized complete block design with three replications. Soil samples were collected before the commencement of the experiment from both sites. The experimental treatments consisted of a control (without fertilizer), chemical, biological and integrated fertilizing systems as follows:

T0: control (no fertilizer application)

T1: chemical fertilizer $(135 \mathrm{~kg} / \mathrm{ha}$ urea fertilizer + $185 \mathrm{~kg} / \mathrm{ha}$ triple superphosphate fertilizer)*

T2: urea chemical fertilizer + phosphorous solubilizing bacteria (Bacillus coagulans Hammer, 1915)

T3: urea chemical fertilizer + mycorrhiza (Glomus intraradices N.C. Schenck \& G.S. Sm.)

T4: urea chemical fertilizer + phosphorous solubilizing bacteria + mycorrhiza
T5: nitrogen fixing bacteria (Sinorhizobium meliloti (Dangeard 1926) De Lajudie et al. 1994, comb. nov) + triple superphosphate fertilizer

T6: nitrogen fixing bacteria + phosphorous solubilizing bacteria

T7: nitrogen fixing bacteria + mycorrhiza

T8: nitrogen fixing bacteria + phosphorous solubilizing bacteria + mycorrhiza

* Chemical fertilizers of triple superphosphate and urea were applied according to soil test to fulfill the requirements of the crop in each site.

Land preparation took place before sowing annual medic in early March. Phosphate solubilizing bacteria (Bacillus coagulans), nitrogen fixing bacteria (Sinorhizobium meliloti), and mycorrhiza (Glomus intraradices) solutions were prepared according to Water and Soil Research Institute of Iran, instructions. After calculating the number of seeds per treatment, the seeds were placed into a polyethylene bag $(30 \mathrm{mg}$ of each inoculation 
substance for $100 \mathrm{~g}$ of seed) along with $4 \%$ arabic gum solution. The seed and the adhesive substance were then gently shaken for 30 seconds. One gram of inoculation substance was added to the adhesive seeds and shaken for 45 seconds, ensuring that the inoculation substance was uniformly distributed among the seeds. The inoculated seeds were spread on an aluminum sheet in shade to dry off.

All experimental plots consisted of 6 planting rows of $25 \mathrm{~cm}$ apart with 5 meters in length. The annual medic 'Robinson' was planted at a rate of $20 \mathrm{~kg}$ of seed per hectare. Seeds were planted in $1 \mathrm{~cm}$ of soil depth. A furrow irrigation system was applied in the irrigated site at four stages as follows: irrigation immediately after planting, at four-leaf, at beginning of flowering and at pod formation stages.

Before sowing the seed, based on soil analysis and according to fertilizer recommendations for annual medic, half of urea fertilizer and all phosphorous fertilizer (in treatments containing phosphorous chemical fertilizer) were applied to the soil in bands. The rest of the nitrogen fertilizer was applied on the corresponding plots when plants reached different growing stages according to the treatments.

After harvesting the plants at each treatment, four soil samples were randomly collected from 0-30 $\mathrm{cm}$ soil depth to evaluate organic matter percent (Walkley and Black, 1934), as well as macro and micro nutrients contents.

Data were analyzed with a three-way analysis of variance (ANOVA) for three factors of location, production condition (i.e. +- irrigation) and fertilizer treatments. Mean treatment values were compared based on the least significant difference (LSD) at 5 percent probability level. SAS and Excel software were used for statistical analysis of experimental data and drawing graphs, respectively.

\section{RESULTS AND DISCUSSION}

\subsection{Soil organic matter and macronutrients content}

The results of ANOVA for soil organic matter content, affected by different fertilizing systems, is shown in Table 3. At both locations, the highest $(1.22 \%)$ and lowest $(0.99 \%)$ soil organic matter percentage was observed in T6 (nitrogen fixing bacteria + phosphorous solubilizing bacteria) and control treatments, respectively (Table 9). The soil organic matter content was significantly increased in T6 fertilizing system indicating that biological fertilizer treatment compared to other fertilizing treatments, provided with better plant growth and consequently higher biomass and organic matter $(\mathrm{OM})$ in the soil. It seems that the continuous chemical fertilizers application in soil, could decrease the availability of soil organic matter due to increasing salt concentration in the soil solution. These processes could consequently reduce the microorganism population colonies. To support this idea we need to continue the experiment for at least few years.

Table 3: The results of ANOVA for soil elements and organic matter

\begin{tabular}{|c|c|c|c|c|c|c|c|c|c|}
\hline 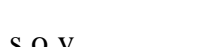 & $\mathrm{df}$ & $\mathrm{OM}$ & $\mathrm{N}$ & $\mathrm{P}$ & $\mathrm{K}$ & $\mathrm{Fe}$ & $\mathrm{Mn}$ & $\mathrm{Cu}$ & $\mathrm{Zn}$ \\
\hline Location (L) & 1 & $* *$ & $* *$ & $* *$ & $* *$ & ns & $* *$ & $* *$ & $* *$ \\
\hline Condition (C) & 1 & $* *$ & $* *$ & $* *$ & $* *$ & $* *$ & $* *$ & $* *$ & $* *$ \\
\hline $\mathrm{L}^{*} \mathrm{C}$ & 1 & ns & ns & $*$ & $* *$ & $* *$ & $* *$ & $* *$ & $* *$ \\
\hline Treatment (T) & 8 & ns & $* *$ & $* *$ & $* *$ & $* *$ & $* *$ & $* *$ & $* *$ \\
\hline $\mathrm{L}^{*} \mathrm{~T}$ & 8 & ns & ns & $* *$ & $* *$ & $* *$ & $\mathrm{~ns}$ & $\mathrm{~ns}$ & $* *$ \\
\hline $\mathrm{C} * \mathrm{~T}$ & 8 & ns & ns & $* *$ & $* *$ & $* *$ & $* *$ & $\mathrm{~ns}$ & $* *$ \\
\hline $\mathrm{L} * \mathrm{C} * \mathrm{~T}$ & 8 & $\mathrm{~ns}$ & ns & $* *$ & $* *$ & $\mathrm{~ns}$ & $* *$ & $\mathrm{~ns}$ & $* *$ \\
\hline
\end{tabular}

ns nonsignificant $*$ Significant at $p \leq 0.05 * *$ Significant at $p \leq 0.01$ 
The highest contents of soil nitrogen $\left(1.31 \mathrm{~g} \mathrm{~kg}^{-1}\right)$, phosphorous (18.3 $\left.\mathrm{mg} \mathrm{kg}^{-1}\right)$ and potassium $\left(42 \mathrm{mg} \mathrm{kg}^{-1}\right)$ were found in integrated fertilizing treatments of $\mathrm{T} 4, \mathrm{~T} 5$ and $\mathrm{T} 6$, respectively. However, the lowest amounts of these elements were found in control treatment (Table 9). It seems that the nitrogen generated from sole chemical fertilizer is more vulnerable to leaching leading to a less plant access, while the integrated biological + chemical fertilizing system, could more effectively increase soil nitrogen due to slow releasing of nutrients. These results are supported by the results of Berger et al., (2013) which indicated that the biofertilizer are more efficient because of slowing down the release of nutrients in rhizosphere.

Table 4: Mean soil organic matter and nutrient content in two farming systems

\begin{tabular}{lcccccccc}
\hline $\begin{array}{l}\text { Experimental } \\
\text { Condition }\end{array}$ & $\begin{array}{r}\mathrm{OM} \\
(\%)\end{array}$ & $\begin{array}{c}\mathrm{N} \\
(\mathrm{g} / \mathrm{kg})\end{array}$ & $\begin{array}{c}\mathrm{P} \\
(\mathrm{mg} / \mathrm{kg})\end{array}$ & $\begin{array}{c}\mathrm{K} \\
(\mathrm{mg} / \mathrm{kg})\end{array}$ & $\begin{array}{c}\mathrm{Fe} \\
(\mathrm{mg} / \mathrm{kg})\end{array}$ & $\begin{array}{c}\mathrm{Mn} \\
(\mathrm{mg} / \mathrm{kg})\end{array}$ & $\begin{array}{c}\mathrm{Cu} \\
(\mathrm{mg} / \mathrm{kg})\end{array}$ & $\begin{array}{c}\mathrm{Zn} \\
(\mathrm{mg} / \mathrm{kg})\end{array}$ \\
\hline Irrigated & $1.07 \mathrm{a}$ & $0.102 \mathrm{~b}$ & $8.89 \mathrm{~b}$ & $366 \mathrm{~b}$ & $4.10 \mathrm{~b}$ & $18.7 \mathrm{a}$ & $1.71 \mathrm{~b}$ & $0.606 \mathrm{~b}$ \\
Dryland & $1.23 \mathrm{a}$ & $0.136 \mathrm{a}$ & $17.5 \mathrm{a}$ & $534 \mathrm{a}$ & $4.40 \mathrm{a}$ & $17.9 \mathrm{a}$ & $2.04 \mathrm{a}$ & $1.06 \mathrm{a}$ \\
\hline LSD (5 \%) & 0.226 & 0.005 & 0.252 & 7.66 & 0.138 & 1.32 & 0.101 & 0.055
\end{tabular}

Means with the same letter in each column are not significantly different at 5 percent probability level.

Table 5: Mean values of organic matter and macronutrient in soil as affected by different fertilizing systems and experimental research sites

\begin{tabular}{|c|c|c|c|c|c|c|c|c|c|c|c|c|}
\hline \multirow{3}{*}{ Treatment $^{\mathrm{a}}$} & \multirow{2}{*}{\multicolumn{2}{|c|}{$\frac{\mathrm{OM}(\%)}{\text { Location }}$}} & \multirow{3}{*}{ Mean } & \multirow{2}{*}{\multicolumn{2}{|c|}{$\begin{array}{l}\mathrm{N}(\%) \\
\text { Location }\end{array}$}} & \multirow{3}{*}{ Mean } & \multirow{2}{*}{\multicolumn{2}{|c|}{$\frac{\mathrm{P}(\mathrm{mg} / \mathrm{kg})}{\text { Location }}$}} & \multirow{3}{*}{ Mean } & \multicolumn{3}{|c|}{$\mathrm{K}(\mathrm{mg} / \mathrm{kg})$} \\
\hline & & & & & & & & & & \multicolumn{2}{|c|}{ Location } & \multirow[t]{2}{*}{ Mean } \\
\hline & $\mathrm{L}^{\mathrm{b}}$ & $\mathrm{L}^{\mathrm{c}}$ & & $\mathrm{L} 1$ & L2 & & $\mathrm{L1}$ & L2 & & $\mathrm{L1}$ & L2 & \\
\hline T0 & 0.855 & 1.15 & $1.00 \mathrm{~b}$ & 0.097 & 0.118 & $0.107 \mathrm{~d}$ & 9.6 & 11.9 & $10.7 \mathrm{e}$ & 370 & 417 & $393 c$ \\
\hline $\mathrm{T} 1$ & 1.00 & 1.27 & $1.03 \mathrm{ab}$ & 0.110 & 0.125 & $0.117 \mathrm{bc}$ & 12.0 & 13.0 & $12.5 \mathrm{~cd}$ & 485 & 439 & $462 \mathrm{ab}$ \\
\hline $\mathrm{T} 2$ & 0.963 & 1.38 & $1.17 \mathrm{ab}$ & 0.110 & 0.123 & $0.116 \mathrm{bcd}$ & 11.6 & 12.3 & $11.9 \mathrm{~d}$ & 455 & 471 & $462 \mathrm{ab}$ \\
\hline $\mathrm{T} 3$ & 0.947 & 1.28 & $1.11 \mathrm{ab}$ & 0.110 & 0.115 & $0.112 \mathrm{~cd}$ & 10.5 & 14.0 & $12.2 \mathrm{~cd}$ & 420 & 478 & $449 b$ \\
\hline $\mathrm{T} 4$ & 0.967 & 1.42 & $1.19 \mathrm{ab}$ & 0.137 & 0.127 & $0.131 \mathrm{a}$ & 12.5 & 13.7 & $13.1 \mathrm{c}$ & 437 & 467 & $451 \mathrm{~b}$ \\
\hline T5 & 1.03 & 1.35 & $1.19 \mathrm{ab}$ & 0.118 & 0.127 & $0.122 \mathrm{abc}$ & 17.7 & 19.0 & $18.3 \mathrm{a}$ & 445 & 451 & $447 b$ \\
\hline T6 & 1.11 & 1.44 & $1.28 \mathrm{a}$ & 0.118 & 0.135 & $0.126 a b$ & 11.5 & 17.4 & $14.4 \mathrm{~b}$ & 475 & 471 & $472 \mathrm{a}$ \\
\hline $\mathrm{T} 7$ & 0.928 & 1.39 & $1.16 \mathrm{ab}$ & 0.110 & 0.133 & $0.121 \mathrm{abc}$ & 13.6 & 11.8 & $12.6 \mathrm{~cd}$ & 465 & 444 & $454 b$ \\
\hline $\mathrm{T} 8$ & 1.04 & 1.16 & $1.10 \mathrm{ab}$ & 0.112 & 0.125 & $0.118 b c$ & 11.2 & 15.2 & $13.1 \mathrm{c}$ & 450 & 470 & $460 \mathrm{ab}$ \\
\hline Mean & $0.984 \mathrm{a}^{\mathrm{d}}$ & $1.32 \mathrm{a}$ & & $0.113 b$ & $0.125 \mathrm{a}$ & & $12.2 \mathrm{~b}$ & $14.2 \mathrm{a}$ & & $444 \mathrm{~b}$ & $456 \mathrm{a}$ & \\
\hline LSD & 0.313 & & & & & & & & & 22.2 & & \\
\hline $\begin{array}{l}\text { local*treat } \\
(5 \%)\end{array}$ & & & & 0.014 & & & 1.487 & & & & & \\
\hline
\end{tabular}

${ }^{\mathrm{a}}$ T0 to T8: Different fertilizing systems, ${ }^{\mathrm{b}}$ Sararoud Experimental Site, ${ }^{\mathrm{c}}$ Mahidash Experimental Site.

${ }^{\mathrm{d}}$ Means with the same letter in each column are not significantly different at 5 percent probability level.

The higher organic matter, nitrogen, phosphorus and potassium contents in the soil of Mahydasht station compared to Sararoud station (Table 9) along with better rainfall distribution in this site, could well explain the higher medic forage yield in this site (Table 2).
The maximum phosphorus content in the soil was observed in the T5 (nitrogen fixing bacteria + triple superphosphate fertilizer) treatment (Table 9). It seems that most of the phosphorous applied as chemical fertilizer to the soil was fixed in a nonabsorbable form (Rodriguez and Reynaldo, 1999). 
Table 6: Mean values of organic matter and micronutrients in soil as affected by different fertilizing systems and Experimental Research Sites

\begin{tabular}{|c|c|c|c|c|c|c|c|c|c|c|c|c|}
\hline \multirow{3}{*}{ Treatment $^{\mathrm{a}}$} & \multicolumn{3}{|c|}{$\mathrm{Fe}(\mathrm{mg} / \mathrm{kg})$} & \multicolumn{3}{|c|}{$\mathrm{Mn}(\mathrm{mg} / \mathrm{kg})$} & \multicolumn{3}{|c|}{$\mathrm{Cu}(\mathrm{mg} / \mathrm{kg})$} & \multicolumn{3}{|c|}{$\mathrm{Zn}(\mathrm{mg} / \mathrm{kg})$} \\
\hline & \multicolumn{2}{|c|}{ Location } & \multirow{2}{*}{ Mean } & \multicolumn{2}{|c|}{ Location } & \multirow{2}{*}{ Mean } & \multicolumn{2}{|c|}{ Location } & \multirow{2}{*}{ Mean } & \multicolumn{2}{|c|}{ Location } & \multirow[t]{2}{*}{ Mean } \\
\hline & $\mathrm{L}^{\mathrm{b}}$ & $\mathrm{L} 2^{\mathrm{c}}$ & & L1 & L2 & & L1 & L2 & & L1 & L2 & \\
\hline T0 & 2.87 & 4.53 & $3.70 \mathrm{~d}$ & 13.7 & 19.4 & $16.5 \mathrm{e}$ & 1.36 & 2.00 & $1.68 \mathrm{~d}$ & 0.367 & 0.637 & $0.501 \mathrm{f}$ \\
\hline $\mathrm{T} 1$ & 3.42 & 5.63 & $4.52 \mathrm{ab}$ & 16.1 & 21.2 & $18.6 \mathrm{c}$ & 1.46 & 2.17 & $1.81 \mathrm{~cd}$ & 0.645 & 0.967 & $0.805 \mathrm{cde}$ \\
\hline $\mathrm{T} 2$ & 3.45 & 4.87 & $4.15 \mathrm{c}$ & 15.6 & 19.6 & $17.5 \mathrm{~d}$ & 1.49 & 2.08 & $1.78 \mathrm{~cd}$ & 0.563 & 0.875 & $0.719 \mathrm{e}$ \\
\hline T3 & 2.85 & 4.63 & $3.74 d$ & 15.5 & 20.6 & $18.0 \mathrm{~d}$ & 1.79 & 2.23 & $2.00 \mathrm{a}$ & 0.807 & 0.902 & $0.854 \mathrm{~cd}$ \\
\hline $\mathrm{T} 4$ & 3.47 & 5.83 & $4.65 \mathrm{a}$ & 14.7 & 20.4 & $17.5 \mathrm{~d}$ & 1.67 & 2.12 & $1.89 \mathrm{abc}$ & 0.608 & 0.852 & $0.730 \mathrm{de}$ \\
\hline T5 & 3.55 & 5.77 & $4.65 \mathrm{a}$ & 18.0 & 23.4 & $20.7 \mathrm{a}$ & 1.58 & 2.25 & $1.91 \mathrm{abc}$ & 0.677 & 1.427 & $1.050 \mathrm{~b}$ \\
\hline T6 & 3.25 & 5.35 & $4.30 \mathrm{bc}$ & 16.0 & 20.7 & $18.3 \mathrm{~cd}$ & 1.62 & 2.05 & $1.83 \mathrm{bc}$ & 0.592 & 0.867 & $0.729 \mathrm{de}$ \\
\hline $\mathrm{T} 7$ & 3.57 & 5.17 & 4.36abc & 17.3 & 21.8 & $19.5 b$ & 1.62 & 2.28 & $1.95 \mathrm{ab}$ & 0.720 & 1.803 & $1.260 \mathrm{a}$ \\
\hline $\mathrm{T} 8$ & 2.92 & 5.42 & $4.16 \mathrm{c}$ & 16.3 & 20.8 & $18.5 \mathrm{c}$ & 1.67 & 2.37 & $2.01 \mathrm{a}$ & 0.802 & 0.972 & $0.889 \mathrm{c}$ \\
\hline Mean $^{\mathrm{d}}$ & $3.25 \mathrm{~b}$ & $5.24 \mathrm{a}$ & & $15.8 \mathrm{~b}$ & $20.8 \mathrm{a}$ & & $1.58 \mathrm{~b}$ & $2.17 \mathrm{a}$ & & $0.642 b$ & $1.03 \mathrm{a}$ & \\
\hline LSD local $*$ treat $(5 \%)$ & 0.435 & & & 1.273 & & & 0.194 & & & 0.178 & & \\
\hline
\end{tabular}

${ }^{\mathrm{a}}$ T0 to T8: Different fertilizing systems, ${ }^{\mathrm{b}}$ Sararoud Experimental Site, ${ }^{\mathrm{c}}$ Mahidash Experimental Site.

${ }^{\mathrm{d}}$ Means with the same letter in each column are not significantly different at 5 percent probability level.

Table 7: Interaction between farming systems and experimental site (location) on organic matter and macronutrient content in soil

\begin{tabular}{|c|c|c|c|c|c|c|c|c|}
\hline \multirow{3}{*}{$\begin{array}{l}\text { Experimental } \\
\text { Condition }\end{array}$} & \multicolumn{2}{|c|}{$\mathrm{OM}(\%)$} & \multicolumn{2}{|c|}{$\mathrm{N}(\%)$} & \multicolumn{2}{|c|}{$\mathrm{P}(\mathrm{mg} / \mathrm{kg})$} & \multicolumn{2}{|c|}{$\mathrm{K}(\mathrm{mg} / \mathrm{kg})$} \\
\hline & \multicolumn{2}{|c|}{ Location } & \multicolumn{2}{|c|}{ Location } & \multicolumn{2}{|c|}{ Location } & \multicolumn{2}{|c|}{ Location } \\
\hline & $\mathrm{L}^{\mathrm{a}}$ & $\mathrm{L}^{\mathrm{b}}$ & L1 & L1 & $\mathrm{L} 2$ & $\mathrm{~L} 2$ & $\begin{array}{ll}\text { L1 } \\
\end{array}$ & L2 \\
\hline $\mathrm{D} 2^{\mathrm{c}}$ & $0.91 \mathrm{~b}$ & $1.05 \mathrm{~b}$ & $0.09 \mathrm{~d}$ & $0.13 b$ & $7.63 \mathrm{~d}$ & $16.84 \mathrm{~b}$ & $331.29 \mathrm{~d}$ & $557.85 \mathrm{a}$ \\
\hline $\mathrm{D} 1^{\mathrm{d}}$ & $1.23 \mathrm{ab}^{\mathrm{e}}$ & $1.41 \mathrm{a}$ & $0.11 \mathrm{c}$ & $0.14 \mathrm{a}$ & $10.15 \mathrm{c}$ & $18.34 \mathrm{a}$ & $401.48 \mathrm{c}$ & $511.29 \mathrm{~b}$ \\
\hline $\begin{array}{l}\text { LSD } \\
\text { Local* Experimental } \\
\text { Condition }(5 \%)\end{array}$ & 0.32 & & 0.007 & & 0.356 & & 10.83 & \\
\hline
\end{tabular}

${ }^{\mathrm{a}}$ Sararoud Experimental Site, ${ }^{\mathrm{b}}$ Mahidash Experimental Site ${ }^{\mathrm{c}}$ Dry farming condition ${ }^{\mathrm{d}}$ Irrigated system

${ }^{\mathrm{e}}$ Means with the same letter in each column are not significantly different at 5 percent probability level.

The interactions between location and production systems showed that the organic matter and macro elements content in the soil under dry farming system were higher than irrigated system (Table 6). The higher yield under the irrigation system could be a good reason of increasing nutrient uptake from the soil (except for potassium) and decreasing the soil nutrients level. The content of extractable potassium before the commencement of the experiment in Sararoud station was more than Mahydasht station, but after annual medic cultivation, the situation was reversed. 
Effect of different fertilizing and ...(Medicago scutellata 'Robinson') on soil organic matter and nutrients status

Table 8: Interaction between farming systems and experimental site (location) on micronutrient content in soil

\begin{tabular}{|c|c|c|c|c|c|c|c|c|}
\hline \multirow{3}{*}{$\begin{array}{c}\text { Experimental } \\
\text { Condition }\end{array}$} & \multicolumn{2}{|c|}{$\mathrm{Fe}(\mathrm{mg} / \mathrm{kg})$} & \multicolumn{2}{|c|}{$\mathrm{Mn}(\mathrm{mg} / \mathrm{kg})$} & \multicolumn{2}{|c|}{$\mathrm{Cu}(\mathrm{mg} / \mathrm{kg})$} & \multicolumn{2}{|c|}{$\mathrm{Zn}(\mathrm{mg} / \mathrm{kg})$} \\
\hline & \multicolumn{2}{|c|}{ Location } & \multicolumn{2}{|c|}{ Location } & \multicolumn{2}{|c|}{ Location } & \multicolumn{2}{|c|}{ Location } \\
\hline & $\mathrm{L1}^{\mathrm{a}}$ & $\mathrm{L} 2^{\mathrm{b}}$ & L1 & L2 & L1 & L2 & L1 & L2 \\
\hline $\mathrm{D} 2^{\mathrm{d}}$ & $3.42 \mathrm{~b}$ & $3.08 \mathrm{c}$ & $17.84 \mathrm{~b}$ & $13.91 \mathrm{c}$ & $1.52 \mathrm{c}$ & $1.63 \mathrm{c}$ & $0.47 \mathrm{c}$ & $0.81 \mathrm{~b}$ \\
\hline $\mathrm{D} 1^{\mathrm{c}}$ & $4.77 b$ & $5.71 \mathrm{a}$ & $19.68 b$ & $22.06 \mathrm{a}$ & $1.89 \mathrm{~b}$ & $2.44 \mathrm{a}$ & $0.73 b$ & $1.32 \mathrm{a}$ \\
\hline $\begin{array}{c}\text { LSD local* } \\
\text { Experimental } \\
\text { Condition }(5 \%)\end{array}$ & \multicolumn{2}{|c|}{1.869} & \multicolumn{2}{|c|}{1.869} & \multicolumn{2}{|c|}{0.143} & \multicolumn{2}{|c|}{0.077} \\
\hline
\end{tabular}

${ }^{\mathrm{a}}$ Sararoud Experimental Site, ${ }^{\mathrm{b}}$ Mahidash Experimental Site ${ }^{\mathrm{c}}$ Dry farming condition ${ }^{\mathrm{d}}$ Irrigated system

Means with the same letter in each column are not significantly different at 5 percent probability level.

Table 9: Interaction of farming systems and fertilizing treatments on organic matter and macronutrient content in soil

\begin{tabular}{|c|c|c|c|c|c|c|c|c|c|c|c|c|}
\hline \multirow{4}{*}{ Treatments } & \multicolumn{3}{|c|}{$\mathrm{OM}(\%)$} & \multicolumn{3}{|c|}{$\mathrm{N}(\%)$} & \multicolumn{3}{|c|}{$\mathrm{P}(\mathrm{mg} / \mathrm{kg})$} & \multicolumn{3}{|c|}{$\mathrm{K}(\mathrm{mg} / \mathrm{kg})$} \\
\hline & \multirow{2}{*}{\multicolumn{2}{|c|}{$\begin{array}{c}\text { Experimental } \\
\text { Condition }\end{array}$}} & \multirow{3}{*}{ Mean } & \multirow{2}{*}{\multicolumn{2}{|c|}{$\begin{array}{c}\text { Experimental } \\
\text { Condition }\end{array}$}} & \multirow{3}{*}{ Mean } & \multirow{2}{*}{\multicolumn{2}{|c|}{$\begin{array}{c}\text { Experimental } \\
\text { Condition }\end{array}$}} & \multirow{3}{*}{ Mean } & \multirow{2}{*}{\multicolumn{2}{|c|}{$\begin{array}{c}\text { Experimental } \\
\text { Condition }\end{array}$}} & \multirow[t]{3}{*}{ Mean } \\
\hline & & & & & & & & & & & & \\
\hline & $\bar{D} 2^{\mathrm{c}}$ & $\mathrm{D} 1^{\mathrm{b}}$ & & $\overline{\mathrm{D} 2}$ & $\overline{\mathrm{D} 1}$ & & D2 & D1 & & D2 & D1 & \\
\hline T0 & 0.89 & 1.08 & 0.99 & 0.09 & 0.10 & 0.09 & 6.80 & 8.15 & 0.09 & 340.83 & 375.00 & 357.91 \\
\hline $\mathrm{T} 1$ & 1.08 & 1.11 & 1.10 & 0.10 & 0.12 & 0.11 & 8.60 & 14.66 & 0.11 & 370.00 & 445.33 & 407.66 \\
\hline $\mathrm{T} 2$ & 1.11 & 1.18 & 1.14 & 0.10 & 0.13 & 0.11 & 7.86 & 16.43 & 0.11 & 360.00 & 554.16 & 457.08 \\
\hline $\mathrm{T} 3$ & 1.05 & 1.23 & 1.14 & 0.09 & 0.13 & 0.11 & 8.43 & 16.06 & 0.11 & 363.33 & 565.83 & 464.58 \\
\hline $\mathrm{T} 4$ & 1.11 & 1.18 & 1.14 & 0.11 & 0.13 & 0.12 & 9.56 & 16.06 & 0.12 & 358.33 & 535.00 & 446.66 \\
\hline $\mathrm{T} 5$ & 1.11 & 1.27 & 1.19 & 0.10 & 0.15 & 0.12 & 15.13 & 16.63 & 0.13 & 375.00 & 545.00 & 460.00 \\
\hline T6 & 1.17 & 1.27 & 1.22 & 0.11 & 0.13 & 0.12 & 8.80 & 21.60 & 0.12 & 390.83 & 520.83 & 455.83 \\
\hline $\mathrm{T} 7$ & 0.99 & 1.39 & 1.19 & 0.10 & 0.13 & 0.11 & 6.71 & 20.10 & 0.11 & 364.16 & 555.00 & 459.58 \\
\hline $\mathrm{T} 8$ & 0.89 & 1.32 & 1.10 & 0.09 & 0.14 & 0.11 & 6.80 & 18.60 & 0.11 & 340.83 & 545.00 & 442.91 \\
\hline Mean & 1.04 & 1.22 & 1.13 & 0.09 & 0.12 & & 0.10 & 0.13 & & 362.59 & 515.68 & \\
\hline \multicolumn{13}{|l|}{ LSD } \\
\hline $\begin{array}{l}\text { FS*FT } \\
(5 \%)^{\mathrm{d}}\end{array}$ & 0.31 & & & 0.01 & & & 1.48 & & & 22.29 & & \\
\hline
\end{tabular}

${ }^{\mathrm{a}} \mathrm{T} 0$ to T8: Different fertilizing systems. ${ }^{\mathrm{b}}$ Dry farming condition ${ }^{\mathrm{c}}$ Irrigated system

${ }^{\mathrm{d}}$ Means with the same letter in each column are not significantly different at 5 percent probability level.

Table 10: Interaction of farming systems and fertilizing treatments on micronutrients content in soil

\begin{tabular}{|c|c|c|c|c|c|c|c|c|c|c|c|c|}
\hline \multirow{4}{*}{ Treatment } & \multicolumn{3}{|c|}{$\mathrm{Fe}(\mathrm{mg} / \mathrm{kg})$} & \multicolumn{3}{|c|}{$\mathrm{Mn}(\mathrm{mg} / \mathrm{kg})$} & \multicolumn{3}{|c|}{$\mathrm{Cu}(\mathrm{mg} / \mathrm{kg})$} & \multicolumn{3}{|c|}{$\mathrm{Zn}(\mathrm{mg} / \mathrm{kg})$} \\
\hline & \multicolumn{3}{|c|}{ Experimental } & \multicolumn{3}{|c|}{ Experimental } & \multirow{2}{*}{\multicolumn{2}{|c|}{$\begin{array}{c}\text { Experimental } \\
\text { Condition }\end{array}$}} & \multirow{3}{*}{ Mean } & \multirow{2}{*}{\multicolumn{2}{|c|}{$\begin{array}{c}\text { Experimental } \\
\text { Condition }\end{array}$}} & \multirow[t]{3}{*}{ Mean } \\
\hline & \multicolumn{2}{|c|}{ Condition } & \multirow[t]{2}{*}{ Mean } & \multicolumn{2}{|c|}{ Condition } & \multirow[t]{2}{*}{ Mean } & & & & & & \\
\hline & $\mathrm{D} 2^{\mathrm{d}}$ & $\mathrm{D} 1^{\mathrm{c}}$ & & D2 & D1 & & D2 & D1 & & D2 & D1 & \\
\hline T0 & 3.40 & 4.46 & 3.93 & 17.11 & 18.76 & 17.94 & 1.58 & 1.91 & 1.74 & 0.41 & 0.62 & 0.51 \\
\hline $\mathrm{T} 1$ & 4.26 & 4.00 & 4.13 & 19.18 & 15.95 & 17.56 & 1.63 & 1.77 & 1.70 & 0.55 & 0.59 & 0.57 \\
\hline $\mathrm{T} 2$ & 4.06 & 4.78 & 4.42 & 18.08 & 18.05 & 18.06 & 1.66 & 1.98 & 1.82 & 0.65 & 1.06 & 0.86 \\
\hline T3 & 3.30 & 4.25 & 3.77 & 17.13 & 17.06 & 17.10 & 1.88 & 1.91 & 1.89 & 0.63 & 0.78 & 0.70 \\
\hline $\mathrm{T} 4$ & 4.48 & 4.18 & 4.33 & 18.11 & 19.00 & 18.55 & 1.76 & 2.13 & 1.95 & 0.63 & 1.07 & 0.85 \\
\hline T5 & 4.71 & 4.81 & 4.76 & 22.10 & 16.93 & 19.51 & 1.67 & 2.01 & 1.84 & 0.54 & 0.82 & 0.68 \\
\hline T6 & 3.86 & 4.60 & 4.23 & 18.21 & 19.33 & 18.77 & 1.62 & 2.15 & 1.88 & 0.60 & 1.56 & 1.08 \\
\hline $\mathrm{T} 7$ & 4.36 & 4.73 & 4.55 & 20.16 & 18.45 & 19.30 & 1.64 & 2.04 & 1.84 & 0.80 & 0.85 & 0.82 \\
\hline $\mathrm{T} 8$ & 3.40 & 4.36 & 3.88 & 17.11 & 18.88 & 18.00 & 1.58 & 2.25 & 1.92 & 0.412 & 1.722 & 1.060 \\
\hline Mean & $3.98 b^{d}$ & $4.46 \mathrm{a}$ & & $18.58 \mathrm{a}$ & $18.04 \mathrm{a}$ & & $1.67 \mathrm{~b}$ & $2.01 \mathrm{a}$ & & $0.58 \mathrm{~b}$ & $1.01 \mathrm{a}$ & \\
\hline \multicolumn{13}{|l|}{ LSD } \\
\hline $\begin{array}{l}\text { FS*FT } \\
(5 \%)\end{array}$ & 0.43 & & 1.27 & & & 19 & & & 0.17 & & & \\
\hline
\end{tabular}

\footnotetext{
${ }^{\mathrm{a}}$ T0 to T8: Different fertilizing systems. ${ }^{\mathrm{c}}$ Dry farming condition ${ }^{\mathrm{d}}$ Irrigated system

${ }^{\mathrm{d}}$ Means with the same letter in each column are not significantly different at 5 percent probability level.
} 
The result of significant interaction of production system and fertilizing treatments is shown in Table 3. The results indicated that for all fertilizing treatments, the phosphorus and potassium concentrations in the dry farming system were consistently higher than in the irrigated system. The lowest phosphorus and potassium content was observed in control treatment under irrigated system. However, the highest amount of phosphorus and potassium in the soil was obtained in T6D1 and T3D1 fertilizing treatments, respectively. Increasing the soil moisture tension subsequently could have reduced nutrients solubility and availability to the plats root. All these findings would be a good answer of the first and second questions of this study.

\subsection{The micronutrients concentration in soil solution}

The results of analysis of variance for the soil micronutrients concentration is shown in Table 3. The effect of location, production systems and their interaction for micronutrients were significant. The results of the interaction of fertilizing system $\times$ location are shown in Table 10. The lowest concentration of micronutrients in both experimental sites was observed in control (no fertilizing treatment). However, the maximum iron content of $4.65 \mathrm{mg} \mathrm{kg}^{-1}$ was observed in $\mathrm{T} 4$ and $\mathrm{T} 5$ fertilizing treatments. The maximum $\mathrm{Mg}$ content of $20.7 \mathrm{ppm}$, copper of $2.01 \mathrm{ppm}$, and $\mathrm{Zn}$ of $1.26 \mathrm{ppm}$ were measured in $\mathrm{T} 5, \mathrm{~T} 8$, and $\mathrm{T} 7$ treatments, respectively.

Overall concentration of the soil micronutrients in Mahydasht was significantly higher than in Sararoud. It should be mentioned that preceding the experiment commencement, the concentration of soil micronutrients in Mahydasht site was already higher than in the Sararoud station (Table 1). The interaction between location and production systems (Table 8) showed a significantly higher concentration of the micronutrients in D1L2 fertilizing treatment.

According to Table 6, fertilizing treatments of T4 and $\mathrm{T} 5$ provided significantly higher iron content compared to other treatments. Application of biological fertilizers along with optimum utilization of chemical fertilizers, played a positive role in maintaining the soil fertility. Our results was supported by other literature reports on the synergistic effects of integrated application of chemical, organic and biological fertilizers on crop yields (Patidar and Mali, 2001; Hariprasad and Niranjana, 2009; Kim et al. 2009). Accordingly the findings of Duarah et al (2011) indicated that the application of biofertilizers (phosphorus solubilize bacteria) can significantly increase the plant biomass compare to application of NPK. Integrated fertilizing systems not only reduces the environmental pollution through reduction in chemical fertilizer consumption, but also improves forage quality features. This is demonstrated that the application of integrated chemical and biofertilizer can improve the soil function to better support the plant nutrition (Mondal et al., 2015; Mal et al., 2013). So the third questions of this study was answered and our finding indicated that the application of biofertilizers (symbiotic bacteria and mycorrhiza) could result in higher yield and quality of annual medic forage. On the other hand this situation would result in the soil nutrients availability and their uptake by plants and that supports the answer of the second question of this study.

According to the results of this study integrated fertilizing system would result in better performance both in soil nutrient and crop yield especially in dry farming conditions. The finding of this research emphasize that the integration of fertilizing systems improved the soil chemical properties.

\section{ACKNOWLEDGMENT}

Special thanks for Dr. Morteza Pourreza from

Razi University for editing manuscript and advice. 


\section{REFERENCES}

Azcon, S. and Kapoor, K. (1998). Effects of inoculation of phosphate solubilizing microorganisms and an arbuscular mycorrizal fungus on mung bean grown under natural soil condition. Mycorrhiza, 7 (5),249253. doi:10.1007/s005720050188

Banerjee, A., Datta, J.K., Mondal, N.K., Chanda, T. (2011). Influence of integrated nutrient management on soil properties of old alluvial soil under mustard cropping system. Commun. Soil Sci. Plant Anal., 42, 2473-2492. doi:10.1080/00103624.2011.609256

Bashan Y. Levanony H. (1990). Current status of Azospirillum inoculation technology: Azospirillum as a challenge for agriculture. Can. J. Microbial, 36, 561-607. doi:10.1139/m90-105

Duarah, I., Deka, M., Saikia, M., Deka Boruah, H.P.(2011). Phosphate solubilizers enhance NPK fertilizer use efficiency in rice and legume cultivation. 3 Biotech, 1, 227-238. doi:10.1007/s13205-011-0028-2

Garai, T.K., Datta, J.K., Mondal, N.K. (2014). Evaluation of integrated nutrient management on Boro rice in alluvial soil and its impacts upon growth, yield attributes yield and soil nutrient status. Arch. Agron. Soil Sci., 60, 1-14. doi:10.1080/03650340.2013.766721

Hariprasad P, Niranjana, S.R. (2009). Isolation and characterization of phosphate solubilizing rhizobacteria to improve plant health of tomato. Plant Soil, 316,13-24. doi:10.1007/s11104-0089754-6

Kim K, Yim WJ, Trivedi P, Madhaiyan M, Deka Boruah HP, Islam MR, Lee G, Sa TM (2009) Synergistic effects of inoculating arbuscular mycorrhizal fungi and Methylobacterium oryzae strains on growth and nutrient uptake of red pepper (Capsicum annum L). Plant Soil. doi:10.1007/s11104-009-0072-4

Mal, B., Mahapatra, P., Mohanty, S., Mishra, H.N. (2013). Growth and yield parameters of okra (Abelmoschus esculentus) influenced by Diazotrophs and chemical fertilizers. Journal of Crop and Weed, 9(2),109-112.
Mondal, T., Datta, J.K., Mondal, N.K. (2015). Chemical fertilizer in conjunction with biofertilizer and vermicompost induced changes in morphophysiological and bio-chemical traits of mustard crop. doi:10.1016/j.jssas.2015.05.001

Olsen, S. R. and Sommers, L. E. (1990). Phosphorus. Pp. 403-431. In: A.S.L. page et al (Eds). Methods of soil Analysis. Part 2.2 and ed., agron. Monogor. 9. ASA, Madison, WL.

Rai, R. (1988). High temperature - adapted A. brasilense strains: growth and interaction response on associative nitrogen fixation, mineral uptake and yield of cheena (Panicum miliaceum) genotype in calcareous soil. J. Agri. Sci. 110, 321-326. doi:10.1017/S0021859600081351

Shabani G.H., Ardakani M.R., Chaichi, M.R, Friedel J.K., Khavazi K.. 2015. Effect of Different Fertilizing Treatments on Nutrient Uptake in Annual Medic (Medicago scutellata cv. Robinson) under Irrigated and Dry Farming Systems. J. Agr. Sci. Tech., 17, (2) 299-310.

Shaoo, R.K., Bharwadj, D., Tuteja., N. (2013). Biofertilizers: a sustainable eco-friendly agricultural approach to crop improvement. In N. Tuteja, and G. Sing (Eds.), Plant Acclimation to Environmental Stress (pp.403-432). Springer Science+Business Media New York. 10013, USA: Springer Science plus Business Media, pp.403-432.

Walkley, A. and Black, C.A. (1934). An examination of the Degtiareff method for the determination of organic matter in soils and proposed modification of the of the chromic acid titration method. Soil Sci. 37, 29-39. doi:10.1097/00010694-19340100000003

Zaidi, A., Khan, M.S and Aamil, M. (2003). Interactive effect of rhizotrophic microorganisms on yield and nutrient uptake of chickpea (Cicer arietinum L.). European Journal of Agronomy, 19,15-21. doi:10.1016/S1161-0301(02)00015-1

Zaidi, A., Khan, M.S and Aamil, M. (2004). Bioassociative effect of rhizospheric microorganisms on yield and nutrient uptake of greengram. Journal of Plant Nutrition, 27, 599-610. doi:10.1081/PLN-120030370 